\title{
Lung neuroendocrine tumors: pathological characteristics
}

\author{
Luisella Righi ${ }^{1}$, Gaia Gatti ${ }^{1}$, Marco Volante ${ }^{1}$, Mauro Papotti ${ }^{2}$ \\ ${ }^{1}$ Department of Oncology, San Luigi Hospital, Orbassano, Italy; ${ }^{2}$ Department of Oncology, City of Health and Science, University of Turin, Torino, \\ Italy \\ Contributions: (I) Conception and design: All authors; (II) Administrative support: M Papotti; (III) Provision of study materials or patients: G Gatti; (IV) \\ Collection and assembly of data: L Righi; (V) Data analysis and interpretation: M Volante; (VI) Manuscript writing: All authors; (VII) Final approval \\ of manuscript: All authors. \\ Correspondence to: Luisella Righi. Department of Oncology, University of Turin, San Luigi Hospital, Regione Gonzole 10, 10043 Orbassano (Torino), \\ Italy. Email: luisella.righi@unito.it.
}

\begin{abstract}
Lung neuroendocrine tumors (NETs) are a heterogeneous family of neoplasms comprising four histologic types, namely typical and atypical carcinoid (TC and AC), large-cell neuroendocrine and small cell carcinoma (SCC). Classification criteria include the number of mitoses per $2 \mathrm{~mm}^{2}$, the occurrence and extent of necrosis, cytological and histological features and immunohistochemistry for neuroendocrine markers. The classification system and the diagnostic workflow of lung NETs are apparently easy to apply and well established. However, several unresolved issues still exist in classification and pathological characterization of these tumors, probably because inter-observer diagnostic reproducibility remains disappointing, likely due to inconsistency in recognizing necrosis, mitoses and cytological details, especially in small biopsy or cytological materials. Furthermore, the lack of strong prognostic and grading criteria leads to the incomplete interpretation of some rare intermediate entities that stand in between AC and large cell neuroendocrine carcinoma (LCNEC) categories.
\end{abstract}

Keywords: Neuroendocrine; carcinoid; classification; grading; small cell carcinoma (SCC)

Submitted Oct 11, 2016. Accepted for publication Dec 26, 2016.

doi: $10.21037 /$ jtd.2017.01.59

View this article at: http://dx.doi.org/10.21037/jtd.2017.01.59

\section{Lung neuroendocrine tumors (NETs): definition and classification}

Lung NETs are a subset of pulmonary neoplasms with shared neuroendocrine differentiation, but with heterogeneous morphological, immunohistochemical and molecular characteristics and considerably different clinical and biological behavior.

They are classified in four major histotypes (1), ranging from the low/intermediate-grade typical carcinoid (TC) and atypical carcinoid (AC) to the high-grade poorly differentiated large cell neuroendocrine carcinoma (LCNEC) and small cell carcinoma (SCC). TC is deemed to be a low-grade malignant tumor with longer life expectation and time to recurrence, AC is an intermediategrade malignant tumor with more aggressive clinical course, somewhat unpredictable clinical behavior and shorter time to recurrence; LCNEC and SCC are high-grade malignant tumors with dismal prognosis, challenging therapy options and, often, difficulties in reliably distinguishing from each other, either pathologically, genetically or clinically (2).

Even if the four histotypes have several different cytological and architectural features, since the World Health Organization (WHO) classification year 2004 (3) [confirmed in the more recent from year 2015 (1)], the differential diagnosis is based on two parameters only: the presence/ absence of necrosis and the mitotic index per $2 \mathrm{~mm}^{2}$. Therefore, a diagnosis of TC is made when the tumor does not show necrosis and the mitotic count is $<2$ mitosis per $2 \mathrm{~mm}^{2}$, the AC group shows up with necrosis and/or a number of mitosis between 2 and 10 per $2 \mathrm{~mm}^{2}$, while high-grade poorly differentiated carcinomas must have $>10$ mitosis per $2 \mathrm{~mm}^{2}$ and they normally present extensive necrotic areas (Figure 1). Cytological features such as cell 


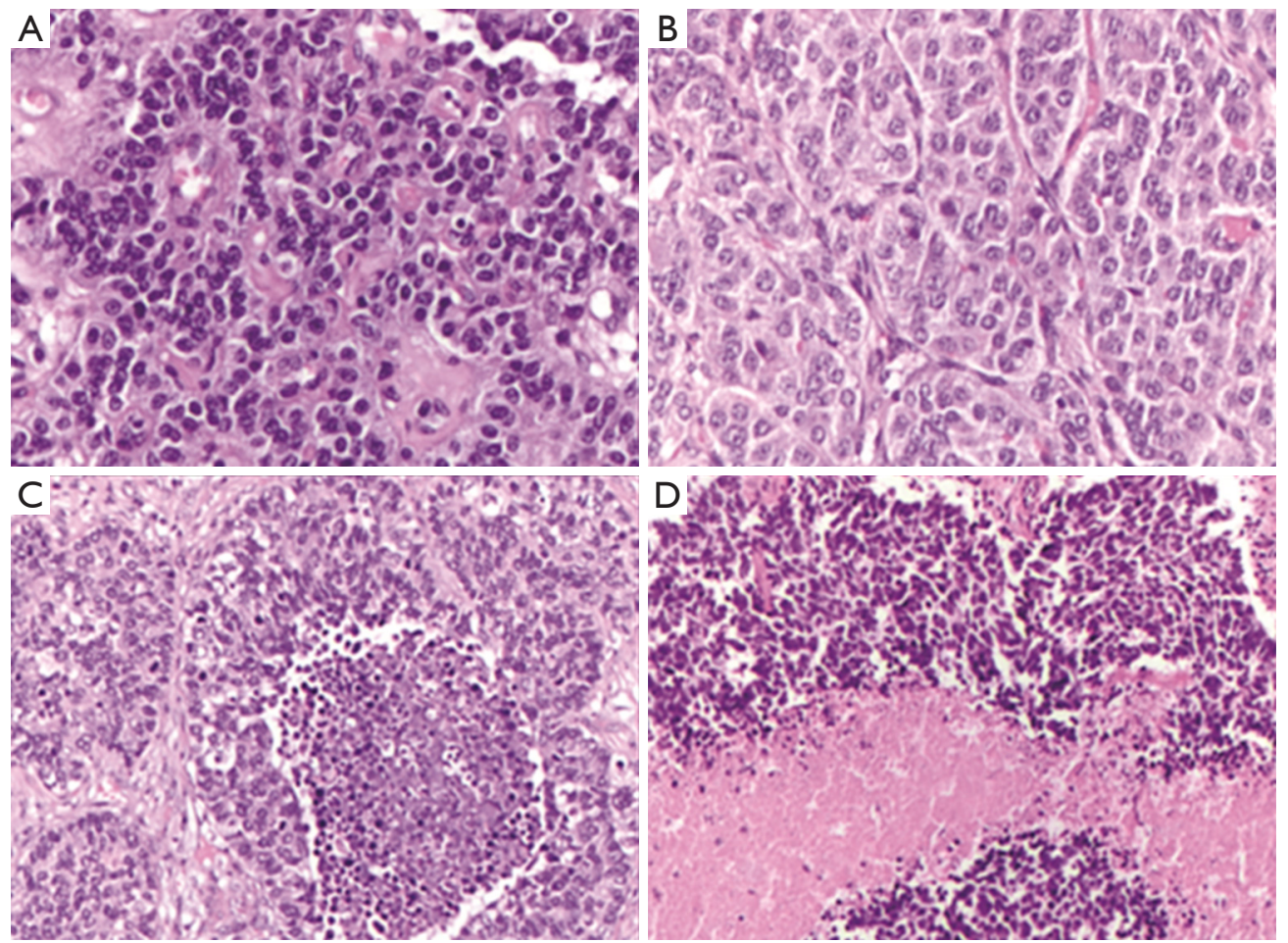

Figure 1 Representative pictures of the four histologic variants of lung neuroendocrine tumors. (A) Typical carcinoid (TC) (ematoxilin \& eosin, 20x); (B) atypical carcinoid (AC) (ematoxilin \& eosin, 20x); (C) large cell neuroendocrine carcinoma (LCNEC) (ematoxilin \& eosin, 10×); (D) small cell carcinoma (SCC) (ematoxilin \& eosin, 10x).

size, nuclear morphology and architecture are additional characteristics useful to distinguish between LCNEC and SCC, but not between AC and TC that always share similar cyto-architectural features.

However, the assessment of the two diagnostic parameters may be affected by high subjectivity (4): the low number of mitoses in a rather large microscopic area, the fact that mitoses are not homogeneously distributed over the tissue section and that they may be confused with apoptotic cells, crushed cells, and granulocytes are proposed to explain this low reproducibility. Furthermore, the distinction between LCNEC and small cell neuroendocrine carcinomas also shows a low reproducibility, due to problems related to sampling procedure and the resulting presence of artifacts, and to the fact that some tumors present transitional cell characteristics between small cell neuroendocrine carcinomas and LCNEC (5-7).

In the previous WHO 2004 (3), the LCNEC category was considered as the neuroendocrine variant of large cell carcinoma (LCC), while the SCC was merely the morphological counterpart of the group of "Non-Small
Cell Lung Carcinoma" (NSCLC). In the last WHO 2015 classification (1) the four histotypes have been gathered together in a separate group of NETs which includes the LCNEC category based on its neuroendocrine morphology and immunoprofile. As a matter of fact, for a diagnosis of NET the demonstration of neuroendocrine differentiation by means of immunohistochemical staining for $\mathrm{NE}$ markers such as chromogranin A and synaptophysin is also required (8) especially in this histotype, to distinguish it from undifferentiated LCC (1).

In lung NETs, there is no specific grading system widely recognized and used, because grading is actually part of histotype definition (1). However, grading systems have been proposed in the literature to better characterize the tumor and enforce the prediction of patients' prognosis. The potential impact of a grading system would mainly affect carcinoids, because LCNEC and SCC are considered high-grade carcinomas by definition and the prognosis is poor, even if there is a small subset of NETs having intermediate features between AC and LCNEC: well differentiated organoid structure resembling carcinoids, 
but with a relatively high proliferative index and invasive ability that partially overlaps the high grade neuroendocrine category in terms of clinical behavior. This subset is not well studied and understood yet, but a grading system would be highly recommended to predict the patient's prognosis and to set new therapeutic options (9). So a grading system for lung NETs should consider different parameters, including both morphology and proliferation index (10) (see below).

Finally, there is no specific staging for lung NETs as they are staged following the AJCC/IASCL TNM staging system also used for non-neuroendocrine lung cancer (11). This staging system results of little significance especially for carcinoids, which are usually less than $3 \mathrm{~cm}$ in diameter, so they normally fit in the lower $\mathrm{T}$ stages. Literature data confirmed a prognostic significance of the TNM classification, but they showed an overlapping survival for carcinoid patients in stages I and II and a significantly different prognosis was observed only for patients in stage III (and IV) as compared to stages I and II. This leads to a limited significance of the staging system for low-/ intermediate-grade lung NETs $(12,13)$. Furthermore, the impact of nodal involvement as a prognostic factor in carcinoids is also controversial in both typical and atypical forms $(12,14)$. In fact, no data have been generated specifically in the group of TC, which in some series indeed present a survival rate significantly higher than that of AC, despite a similar rate of cases with positive nodal status (15).

Some other pathological characteristics have been analyzed as potential predictors of biological and clinical behavior in lung neuroendocrine neoplasms, with special reference to carcinoids (16). Blood and lymphovascular invasion, invasion of the lung parenchyma or cartilage has been also associated to AC histotypes and poor prognosis (17). However, most of the features above mentioned have been investigated in single studies lacking multivariate statistical analysis, and are frequently associated to the AC histotypes.

\section{The role of $\mathrm{Ki}-67$}

The role of the Ki-67 antigen in lung NET has been widely studied $(6,9,10)$, with potential diagnostic, prognostic and grading implications emerging from several independent investigations (10). A diagnostic role has been denied, although Ki-67 labeling index (LI) is diversely ranked among the four histological variants $(1,10)$, likely due to some overlap of biologically similar tumor categories (10). Currently, the only diagnostic role of Ki-67 LI in lung NET regards the separation of TC/AC from SCC on cytology and/or biopsy samples, especially if scarce material and/or crush artifacts are present (1). On the other hand, $\mathrm{Ki}-67$ proliferation index has been demonstrated to be a strong prognostic indicator in lung NETs, even if some authors demonstrated that Ki-67 alone has a limited role in predicting short-term overall survival (3). Finally a role of $\mathrm{Ki}-67 \mathrm{LI}$ in tumor grading is a controversial issue. In fact tumor grading in lung NET is carried out by means of histology, according to which TC, AC and LCNEC/ SCC are used to indicate low-grade, intermediate-grade and high-grade tumors, respectively (1). However, the proportion of lung NETs with similar histology but different behavior is clinically important, as to justify a grading system complementary to conventional terminology (10). Regardless of the real nature of such thoracic carcinoids with a relatively high $\mathrm{Ki}-67$ index, indeed the use of $\mathrm{Ki}-67$ is not officially required for the classification of thoracic NETs, although the recent WHO classification mentions that Ki-67 index might have a role in stratifying neuroendocrine lung tumors (1). A recent study of almost 400 pulmonary NETs (10) proposed a grading system that combined morphological parameters (mitotic count and necrosis) with the Ki-67 index. By combining mitoses, necrosis and Ki-67 index thresholds, a grading system (G1 to G3) was generated based on the occurrence of at least two of three parameters meeting the required cut-off levels. At the histological level, all TC resulted to be G1 while among 75 AC, 45 were attributed to G2 (the remaining 29 being down-graded to G1 and 1 up-graded to G3). In the poorly differentiated group, 78 of 86 LCNEC and 76 of 82 SCC were confirmed G3, however 8 and 6 , respectively, were down-graded to G2. Importantly, AC were split into all the three tumor grades reflecting the inherent behavioral heterogeneity of AC, some of which behave very similarly to TC, whereas others follow a much more aggressive, not too different from that of poorly differentiated lung NETs. Of note, this multiparametric grading system approach turned out to be an accurate predictor of lung NET behavior. The combined assessment of these three parameters outperformed each individual parameter in predicting patient overall survival, showing minimal overlap of $95 \%$ confidence intervals among the three defined categories. Certainly many efforts are needed for validating this grading proposal in lung NET, by accruing independent series of resected tumor specimens, as well as for setting up a reliable grading system in small samples, which are often the only available material at the time of the initial diagnosis or in tumor metastases, where tumor grading might bear 


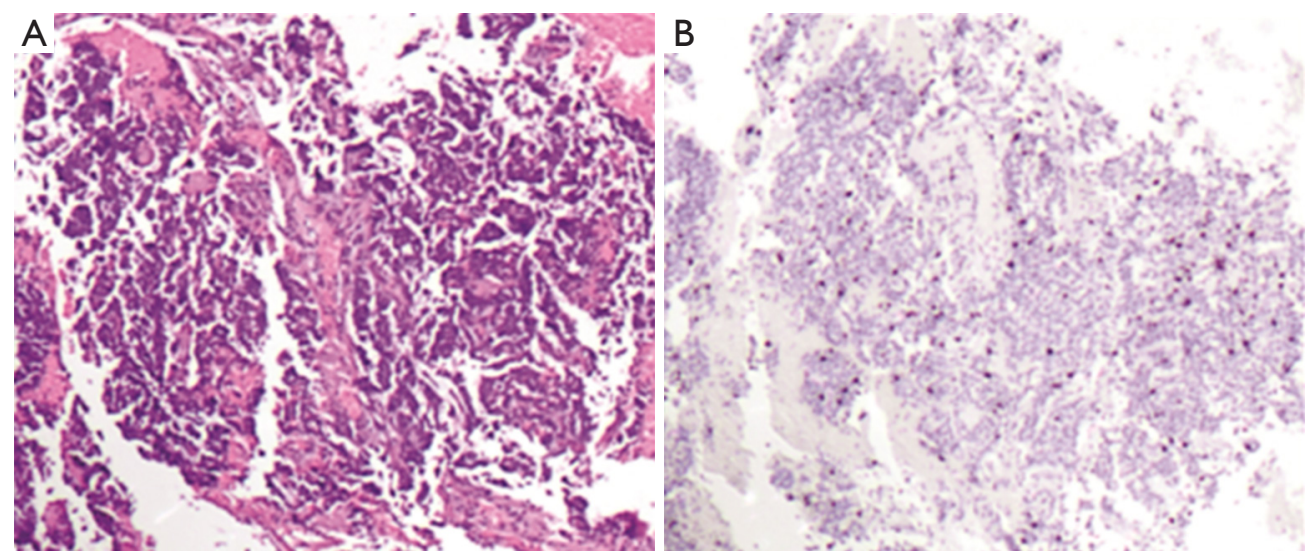

Figure 2 Pictures of a bioptical sample of lung neuroendocrine tumor. (A) Bronchial biopsy sample with low grade neuroendocrine tumor with no necrosis, no mitosis and neuroendocrine morphology (ematoxilin \& eosin, 4x); (B) Ki-67 staining showed an intermediate proliferative activity (LI =10\%) (immunostaining, 4×).

clinical relevance (8).

Another issue to be solved by pathologist is the method for quantifying Ki-67 LI, either manual or by means of automated systems. Few methodological studies addressed the point of Ki-67 LI quantification in surgical specimens of lung NET $(6,10,18-20)$. This is probably due to the irrelevant role of $\mathrm{Ki}-67$ in diagnosis, prognosis, and grading of these tumors, which are still evaluated by morphology, only. There are different options to quantify Ki-67 antigen in lung NET, traditionally carried out by immunohistochemistry using the MIB-1 clone and scoring the percentage of positive tumor cells (LI), i.e., manual counting, digital image analysis or eyeball estimation $(21,22)$. In a recent personal study (manuscript submitted) we provide different methodological criteria for assessing Ki-67 LI in biopsy samples of lung NET by comparing expected results for paired surgical specimens. In particular, we demonstrated that, once hot spot regions (HSR) were identified in either pre-surgical biopsies or surgical specimens, potential discrepancies due to sampling, sizing or intra-tumor heterogeneity of $\mathrm{Ki}-67$ antigen distribution were accounted for by counting 2,000 cells, $2 \mathrm{~mm}^{2}$-spanning HSR(s) or the entire biopsy fragment(s), regardless of histology. More specifically, Ki-67 LI provided the same range of information in biopsy samples as in surgical specimens, thus allowing low- to intermediategrade and high-grade tumors to be accurately separated by using a $20 \%$ cut-off threshold. On the contrary, necrosis and mitosis failed to reach similarly reliable results. This methodology study paves the way to utilize Ki-67 LI in a clinical setting of metastatic lung NET, as an operational criterion for decision-making processes.

\section{Diagnosis on small biopsies and cytological material: a practical approach}

In lung NET classification, defining criteria have been established using surgical specimens, whereas biopsies of lung NET continue to remain a methodologically poorly explored tumor burden with non-trivial clinical implications. However, an increasing number of first diagnoses are made on cytological material (sputum, bronchial aspiration, trans-bronchial needle aspiration, fine needle aspiration from superficial lesions and/or metastases) or small biopsies (trans-bronchial, core biopsy etc.). In small specimens, indeed crush artifacts, necrosis or scarce representative material may lead to a potentially inconclusive diagnosis due to the intrinsic features of the submitted sample. These limits regard particularly the distinction between low-intermediate carcinoids (TC and AC) because, as mentioned above, even if $\mathrm{Ki}-67 \mathrm{LI}$ is not mandatory for diagnosis, it may be useful in distinguishing carcinoids from high-grade carcinomas, only (23) (Figure 2).

So when facing a lung biopsy with suspected NET lesion, after a metastasis from NET of other sites is excluded, the following rules should be followed.

If a high grade morphology, extensive necrosis and high mitotic index are present, a diagnosis of "high grade neuroendocrine carcinoma" should be made with the specification of large or small cells whenever possible. 
In this case, the $\mathrm{Ki}-67 \mathrm{LI}$ is usually $>50 \%$ and it is not mandatory for the diagnosis, although it could probably be useful for the treatment decision.

On the other hand, in case of a low grade tumor with neuroendocrine morphology and phenotype, a diagnosis of $\mathrm{AC}$ is possible only if focal necrosis or mitotic rate from 2 to 9 are recorded; on the other hand (no necrosis, 1 or no mitosis) a diagnosis of "neuroendocrine neoplasia" or "carcinoid tumor" should be made. In this case, Ki-67 LI should be useful for a possible differential diagnosis as $1-2 \%$ is usual in TC while a $\mathrm{Ki}-67$ from $4 \%$ to $15 \%$ is usual in $\mathrm{AC}$; as opposed to high values that directly point to a SCC (23).

\section{Conclusions}

Lung NETs are a quite heterogeneous group of human malignancies with profound differences in the pathologic and behavioral characteristics. Such heterogeneity of some lung AC and LCNEC, and the proven clinical utility of tumor grading in other neuroendocrine neoplasms support the generation of such a grading also in the lung. To this regard, no single parameter is sufficient to predict behavior (neither the sole morphology nor Ki-67 index), however a combined system may derive an accurate grading system of potential prognostic stratification and therapeutic usefulness. While it is not possible to distinguish the four categories of lung NET in biopsy samples, where only carcinoid, SCC and, putatively, LCNEC can be morphologically recognized, Ki-67 LI may be helpful, whenever clinical reasons require a clarification for prognosis or treatment options in individual metastatic lung NET patients. The final goal is to unravel the inherent complexity of lung NET to finally increase our options of therapy in these tumor patients.

\section{Acknowledgements}

None.

\section{Footnote}

Conflicts of Interest: The authors have no conflicts of interest to declare.

\section{References}

1. Travis WD, Brambilla E, Burke AP, et al. WHO Classification of Tumours of the Lung, Pleura, Thymus and Heart. World Healt Organization Classification Tumours. Fourth edition. Lyon: IARC Press, 2015.

2. Pelosi G, Fabbri A, Cossa M, et al. What clinicians are asking pathologists when dealing with lung neuroendocrine neoplasms? Semin Diagn Pathol 2015;32:469-79.

3. Travis WD, Brambilla E, Müller-Hermelink HK, et al. World Health Organization Classification of Tumours. Pathology and Genetics of Tumours of the Lung, Pleura, Thymus and Heart. World Healt Organization Classification Tumours 7. Lyon: IARC Press, 2004.

4. Volante M, Gatti G, Papotti M. Classification of lung neuroendocrine tumors: lights and shadows. Endocrine 2015;50:315-9.

5. Skov BG, Krasnik M, Lantuejoul S, et al. Reclassification of neuroendocrine tumors improves the separation of carcinoids and the prediction of survival. J Thorac Oncol 2008;3:1410-5.

6. Swarts DR, van Suylen RJ, den Bakker MA, et al. Interobserver variability for the $\mathrm{WHO}$ classification of pulmonary carcinoids. Am J Surg Pathol 2014;38:1429-36.

7. den Bakker MA, Willemsen S, Grünberg K, et al. Small cell carcinoma of the lung and large cell neuroendocrine carcinoma interobserver variability. Histopathology 2010;56:356-63.

8. Caplin ME, Baudin E, Ferolla P, et al. Pulmonary neuroendocrine (carcinoid) tumors: European Neuroendocrine Tumor Society expert consensus and recommendations for best practice for typical and atypical pulmonary carcinoids. Ann Oncol 2015;26:1604-20.

9. Pelosi G, Pattini L, Morana G, et al. Grading lung neuroendocrine tumors: Controversies in search of a solution. Histol Histopathol 2017;32:223-41.

10. Rindi G, Klersy C, Inzani F, et al. Grading the neuroendocrine tumors of the lung: an evidence-based proposal. Endocr Relat Cancer 2013;21:1-16.

11. Travis WD, Giroux DJ, Chansky K, et al. The IASLC Lung Cancer Staging Project: proposals for the inclusion of broncho-pulmonary carcinoid tumors in the forthcoming (seventh) edition of the TNM Classification for Lung Cancer. J Thorac Oncol 2008;3:1213-23.

12. Filosso PL, Oliaro A, Ruffini E, et al. Outcome and prognostic factors in bronchial carcinoids: a single-center experience. J Thorac Oncol 2013;8:1282-8.

13. Daddi N, Schiavon M, Filosso PL, et al. Prognostic factors in a multicentre study of 247 atypical pulmonary carcinoids. Eur J Cardiothorac Surg 2014;45:677-86.

14. Johnson R, Trocha S, McLawhorn M, et al. Histology, not lymph node involvement, predicts long-term survival in 
bronchopulmonary carcinoids. Am Surg 2011;77:1669-74.

15. Ferolla P, Daddi N, Urbani M, et al. Tumorlets, multicentric carcinoids, lymph-nodal metastases, and longterm behavior in bronchial carcinoids. J Thorac Oncol 2009;4:383-7.

16. Tsuta K, Raso MG, Kalhor N, et al. Histologic features of low- and intermediate-grade neuroendocrine carcinoma (typical and atypical carcinoid tumors) of the lung. Lung Cancer 2011;71:34-41.

17. Ha SY, Lee JJ, Cho J, et al. Lung parenchymal invasion in pulmonary carcinoid tumor: an important histologic feature suggesting the diagnosis of atypical carcinoid and poor prognosis. Lung Cancer 2013;80:146-52.

18. Costes V, Marty-Ané C, Picot MC, et al. Typical and atypical bronchopulmonary carcinoid tumors: a clinicopathologic and KI-67-labeling study. Hum Pathol 1995;26:740-5.

19. Warth A, Fink L, Fisseler-Eckhoff A, et al. Interobserver agreement of proliferation index (Ki-67) outperforms

Cite this article as: Righi L, Gatti G, Volante M, Papotti M. Lung neuroendocrine tumors: pathological characteristics. J Thorac Dis 2017;9(Suppl 15):S1442-S1447. doi: 10.21037/ jtd.2017.01.59 mitotic count in pulmonary carcinoids. Virchows Arch 2013;462:507-13.

20. Walts AE, Ines D, Marchevsky AM. Limited role of Ki-67 proliferative index in predicting overall short-term survival in patients with typical and atypical pulmonary carcinoid tumors. Mod Pathol 2012;25:1258-64.

21. Pelosi G, Rindi G, Travis WD, et al. Ki-67 antigen in lung neuroendocrine tumors: unraveling a role in clinical practice. J Thorac Oncol 2014;9:273-84.

22. Tang LH, Gonen M, Hedvat C, et al. Objective quantification of the Ki67 proliferative index in neuroendocrine tumors of the gastroenteropancreatic system: a comparison of digital image analysis with manual methods. Am J Surg Pathol 2012;36:1761-70.

23. Pelosi G, Rodriguez J, Viale G, et al. Typical and atypical pulmonary carcinoid tumor overdiagnosed as small-cell carcinoma on biopsy specimens: a major pitfall in the management of lung cancer patients. Am J Surg Pathol 2005;29:179-87. 